prevalences were reported among 16-19 year olds for CT 13\% (95\% CI; 10.8-16.4), NG, 12\% (95\% CI; 9.7-15.1) and TV, 17\%(95\% CI; 13.7-21.1). There were 17,848 STI tests conducted in 2010 and among females aged 16-34; 33.3\% had $\geq 1$ STI (highest in 16-19 year olds: $48.9 \%$ ) and $21.3 \%$ of males had $\geq 1$ STI (highest in 16-19 year olds:33.4\%). The most frequent co-infection was CT and NG which was found in $3.4 \%$ of females (highest in 16-19 year olds: $8.6 \%$ ) and $3.9 \%$ of males (highest in 16-19 year olds:10.1\%).

Discussion STRIVE has provided information not previously available in regard to a comprehensive epidemiological picture of STI morbidity and health service responses in remote Aboriginal communities and highlights work required especially among young people. The results of STRIVE may be of relevance to other areas globally with STI endemic rates.

\section{P6.008 EXTERNAL QUALITY ASSURANCE WITH DRIED TUBE SPECIMENS (DTS) FOR POINT OF CARE SYPHILIS AND HIV TESTS: EXPERIENCE IN AN INDIGENOUS POPULATIONS SCREENING PROGRAMME IN THE BRAZILIAN AMAZON}

doi:10.1136/sextrans-2013-051184.1162

'A S Benzaken, ${ }^{2} \mathrm{M}$ Bazzo, 'E Galban, ${ }^{1}$ P Pinto, ${ }^{2} \mathrm{C}$ L Nogueira, ${ }^{2} \mathrm{~L}$ Golfetto, ${ }^{3} \mathrm{~N}$ S Benzaken, ${ }^{4} \mathrm{~K}$ Sollis, ${ }^{4} \mathrm{D}$ Mabey, ${ }^{4} \mathrm{R}$ Peeling. ${ }^{1}$ Alfredo da Matta Foundation, Manaus, Brazil; ${ }^{2}$ Federal University of Santa Catarina, Florianópolis, Brazil; ${ }^{3}$ Nilton Lins University, Manaus, Brazil; ${ }^{4}$ London School of Hygiene and Tropical Medicine, London, UK

Background The availability of point of care(POC) tests for infectious diseases has revolutionised the provision of health care for remote rural populations without access to laboratories. However, little attention has been given to quality assurance for POC tests. In a screening project that tested 45,226 adults of both sexes by 268 Health Care Workers(HCWs), in remote indigenous populations in the Amazon region of Brazil, where the overall prevalence of syphilis was $1.6 \%$, and of HIV 0.1\%, we evaluated the use of Dry Tube Specimens(DTS) for External Quality Assurance(EOA) for POC HIV and Syphilis tests. Methods The EOA programme was implemented from March 2010 to March 2011 using DTS panels developed by a reference laboratory, containing samples with negative and positive results at different antibody concentrations, for HIV and Syphilis infection. These were re-suspended and tested in the communities by each HCW. We also conducted stability tests for the panels at the reference laboratory.

Results Results from $268 \mathrm{HCWs}$, responsible for implementing the POC tests at six Indigenous District(DSEI) participated in the EOA programme, showed a concordance rate of $90 \%$ for syphilis and $93 \%$ for HIV (Kappa coefficients of 0.74 and 0.78 respectively) with reference laboratories for a total of 1,608 determinations. The highest rate of inaccurate diagnoses occurred in positive samples of very low antibody concentration (40\% for syphilis and $11.9 \%$ for HIV). The stability tests showed that titers were stable for up to one week at $30^{\circ} \mathrm{C}$ in dry conditions.

Conclusion The results show that errors in the interpretation of POC test results were identified by the EOA programme using DTS. The use of POC tests for syphilis and HIV is now recommended as a policy by the Brazilian government. EOA/using DTS can help to improve the quality of these screening programmes and is already being implemented nationally.

\section{P6.009 IMPROVED TIMELY DIAGNOSIS OF HIV RELATED TO THE POLICY OF EXPANDING ACCESS TO DIAGNOSIS IN BRAZIL}

doi:10.1136/sextrans-2013-051184.1163

H Bernal, A A Pereira, A Pires, A R Costa, E Z Ayer, A Bolzan, M R Arruda, A I Sousa, A P Pascom, M A Freitas. Ministry of health, Brasilia, Brazil

Improved timely diagnosis of HIV related to the policy of expanding access to diagnosis in Brazil.
Background In the last years the Brazilian Ministry of Health expanded the use of rapid tests (RT) for HIV. RT is especially useful for pregnant women, vulnerable populations, people with limited access to health services and at national testing campaigns. This policy aims to ensure timely diagnosis and treatment, what will impact directly on morbimortality of people living with HIV/aids (PLWHA).

Methods National network consists of 90 national public laboratories responsible for performing CD4 count for the public health system. Between 2005 and 2012, 3.7 million tests were registered in its database. Based on the first CD4 count registered, diagnosis was classified as very late $\left(<200\right.$ cells $\left./ \mathrm{mm}^{3}\right)$, late (between 200 and 350 cells $/ \mathrm{mm}^{3}$ ) and timely $\left(>350\right.$ cells $\left./ \mathrm{mm}^{3}\right)$.

Results Our database was comprised of 321 thousand PLWHA > 18 y.o. who started on follow up in the public health system between 2005 and 2012. In 2005, 30.6\%, 21.6\% and 47.7\% of PLWHA were diagnosed very late, late and timely, respectively. In 2012 there was a significant decrease in very late and late diagnosis and an increase in timely diagnosis: 29\%, 18\% and 53\% respectively. Conclusion The policy of expanded access to rapid test certainly contributed to these results. Indeed, there was an increase in rapid tests supply: from 509,180 in 2005 to 3,750,000 in 2012, facilitating that PLWHA are diagnosed with higher CD4 counts, what is confirmed by the increase of median first CD 4 count from 335 in 2005 to 365 cells $/ \mathrm{mm}^{3}$ in 2012 .

\section{P6.010 IMPLEMENTATION OF VIA FOR CERVICAL CANCER SCREENING IN A SEXUALLY TRANSMITTED INFECTION CLINIC IN LILONGWE, MALAWI}

doi:10.1136/sextrans-2013-051184.1164

1,2M Hosseinipour, 'B Ndalama, 1,2N E Rosenberg, 'G Kamanga, ${ }^{1} \mathrm{C}$ Mapanje, ${ }^{3} \mathrm{~S}$ Phiri, ${ }^{2}$ W C Miller, ${ }^{1}, 2 \mathrm{~F}$ Martinson, ${ }^{1,2} \mathrm{I}$ Hoffman. ${ }^{1}$ UNC Project, Lilongwe, Malawi; ${ }^{2}$ University of North Carolina, Chapel Hill, NC, United States; ${ }^{3}$ Lighthouse Trust, Lilongwe, Malawi

Background Cervical cancer is the most common female cancer in Malawi with high mortality. Cervical cancer can be averted if precancerous lesions are detected early and treated. Visual Inspection with Acetic Acid (VIA) is an effective screening method for resourcelimited settings. In an STI Clinic in Lilongwe, Malawi, VIA screening implementation was assessed through the NIH-funded Medical Education Partnership Initiative. In this setting, 20\% of women have reactive VIA results.

Methods Females attending the Kamuzu Central Hospital STI Clinic from October 2012 to January 2013 were included. Screening was recommended for women $25-45$ years and women $<25$ years at clinician discretion. We explored the proportion of women who were screened, characterised write-in reasons for non-screening, and conducted binomial regression to explore screening predictors.

Results During this 3.5 month period, 956 women presented for 1240 STI clinic visits. Four percent of women $<25$ and $19 \%$ of women 25-45 received VIA screening. Among women 25-45, common reasons for not screening included postponement (19\%) (often due to STI treatment or cervical pain), recent screening (14\%), menses $(8 \%)$, and pregnancy (3\%). Few refused (3\%). Many did not have reasons recorded (44\%). Screening was less common among women presenting through partner-referral $(0.3$; CI $0.1,1.1)$ and among women who did not receive pelvic exams as part of STI assessment (0.6, CI: 0.3, 0.9).

Conclusions In this high risk setting, VIA implementation was feasible and acceptable. On the day of presentation, many women were not screened due to cervical pain, STI treatment, menses, or pregnancy. Ensuring that these women return for screening is important. Offering VIA to all women is essential, even those not otherwise receiving a pelvic exam. Clear guidelines on whether and 
when to screen women $<25$ years are needed. Such measures will help detect precancerous lesions and avert cervical cancer.

\section{P6.011 SURE START IN PUNE, INDIA: THE CONVERGENCE MODEL OF MATERNAL AND NEWBORN HEALTH AND HIV}

doi:10.1136/sextrans-2013-051184.1165

S Shankar Wunnava, L M Menezes. PATH, New Delhi, India

Background The Sure Start project is a seven year initiative implemented by PATH to improve maternal and newborn health and save lives in India. In Pune, Maharashtra, Sure Start focused on reducing maternal and newborn mortality rates and HIV among pregnant women, including the additional health care needs of HIV positive pregnant women. The objective was to raise awareness of HIV among pregnant women and motivate them to undergo HIV testing and to test the feasibility of convergence of HIV/AIDS and $\mathrm{MNH}$ for synergy in impact. Monitoring of Maternal and Newborn Status (MOMS) committees were involved for regular monitoring and follow-up in the community.

Methods A quasi-experimental study was conducted with preand post-intervention surveys, without a comparison group. Quantitative methods were used to capture changes associated with implementation of a Common Minimum Package and qualitative methods to assess model specific changes.

Results Percent of women who received three or more antenatal checkups increased from $83 \%$ to $97 \%$ from baseline to end-line, the percent of women initiating breastfeeding within one hour increased from $47 \%$ to $52 \%$ during the same period. Percent of women having institutional deliveries increased from $84 \%$ to $95 \%$. Percent of mothers who visited a health facility for a checkup during the postnatal period increased from $49 \%$ to $73 \%$. $92 \%$ of women had heard about HIV/AIDS, among these women 69\% knew about medication available to reduce the risk of transmission to a baby. Of $94 \%$ of those who knew about HIV/AIDS, $83 \%$ had undergone testing. Of the women advised to go for HIV testing $51 \%$ reported that flash cards used by Sure Start provided information about HIV.

Conclusion The model demonstrated the importance of MOMS committees in creating awareness and expanding knowledge. A convergence model helps in mainstreaming HIV positive women to avail of quality $\mathrm{MNH}$ care.

\section{P6.012 STI SCREENING AT HIV TREATMENT CENTRES FOR MEN WHO HAVE SEX WITH MEN CAN BE COST-EFFECTIVE}

doi:10.1136/sextrans-2013-051184.1166

${ }^{1,2}$ H J Vriend, ${ }^{1} A$ K Lugnér, ${ }^{1} M$ Xiridou, SSHIM (STI screening HIV-infected MSM) Working Group. 'National Institute for Public Health and the Environment (RIVM), Bilthoven, The Netherlands; '2Academic Medical Center (AMC), Amsterdam, The Netherlands

Background For HIV-infected men who have sex with men (MSM), implementation of routine STI screening into care at HIV treatment centres can substantially reduce STI incidence and possibly HIV incidence in the MSM population. Some countries already combine STI and HIV care, but its cost-effectiveness has not been explored. This study was designed to estimate the cost-effectiveness of providing routine anorectal chlamydia screening to MSM in care at HIV treatment centres in the Netherlands, where STI and HIV care are not currently combined.

Method Outcomes of a transmission model describing the sexual transmission of HIV and chlamydia in MSM over a 20 -year period were used as input for an economic model. Inclusion of multiple STIs were not allowed due to the complexity of the transmission model. The incremental cost-effectiveness ratio (ICER) was calculated for four scenarios: once- and twice-yearly routine chlamydia screening at HIV treatment centres among MSM who do/do not seek screening elsewhere.

Results Costs will be saved by routine chlamydia screening of MSM in care at HIV treatment centres if these patients seek little or no screening elsewhere. Opportunistic screening is considerably more expensive than routine screening offered within a scheduled visit. Adding once-yearly chlamydia screening for MSM in care at HIV treatment centres is cost-saving when $30 \%$ or fewer of those men seek once-yearly screening elsewhere. Twice-yearly routine screening at HIV treatment centres is cost-effective only when no opportunistic screening takes place.

Conclusion Adding annual chlamydia screening to the HIV consultation will be cost-saving as long as only a limited proportion of men are opportunistically screened. The ICER was most sensitive to the percentage of MSM that continue to be screened elsewhere.

\section{P6.013 RAPID HIV DIAGNOSTIC TESTING IN BRAZIL AND ITS CONTRIBUTION TOWARD REDUCING VERTICAL TRANSMISSION OF HIV AND SYPHILIS}

doi:10.1136/sextrans-2013-051184.1167

E Ayer, A Mello, A Bolzan, D Serafim, E Santos, L Pittaluga, S Vivaldini. Ministry of Health - Brasil, Brasilia, Brazil

Background In 2012, Brazil's Ministry of Health (MoH), via its Department of STDs, AIDS and Viral Hepatitis, increased the availability of rapid testing for HIV diagnosis and syphilis screening by means of a government programme called Rede Cegonha (the Stork Network), allowing for the strengthening of actions to promote, prevent, and assist STD/AIDS patients in the primary care network by servicing pregnant women during their prenatal visits, with the aim of reducing vertical transmission of HIV and syphilis.

Methods The $\mathrm{MoH}$ purchases and distributes rapid tests (RTs) produced domestically. The process was initiated by sensitising local managers to the need for implementing RTs in primary care units. Healthcare professionals were selected and trained to conduct RTs and to provide counselling. The RTs were delivered and introduced into the service routine in order to assist pregnant women and their sexual partners.

Results 27 states adhered to the programme, with 2,500 municipalities adopting the methodology and a total of 1,031 professionals being trained. In 2012, 3.7 million RTs for HIV and 1.1 million RTs for syphilis were distributed.

Conclusion The government's directive expedited the implementation process and the involvement of sanitary authorities. Rapid tests were found to be an agile and complementary tool in expanding access to diagnosis and speeding up the care needed to prevent and reduce vertical transmission of HIV and syphilis. This was an opportunity to update professionals and improve their approach, making it possible to provide integral care to pregnant women, their partners, and newborns.

\section{P6.014 KENYA'S PROGRESS TOWARDS ELIMINATION OF MOTHER- TO-CHILD TRANSMISSION (EMTCT) OF HIV}

doi:10.1136/sextrans-2013-051184.1168

'M A Mudany, ${ }^{2} \mathrm{M}$ Sirengo, 'L W Nganga, ${ }^{3} \mathrm{~A}$ Gichangi. ${ }^{1}$ Centers for Disease control and prevention, Nairobi, Kenya, ${ }^{2}$ National AIDS and STI Control Program (NASCOP), Nairobi, Kenya, ${ }^{3}$ Centers for Disease Control and prevention (CDC), Nairobi, Kenya

Background According to Kenya's Demographic Health Survey (KDHS) 2009, 6.2\% of 39 million residents are HIV-infected. Of 1.5 million annual pregnancies, 93,000 are HIV-infected. Without interventions, $35-40 \%$ of their infants would be infected. We describe Kenya's progress towards eMTCT. 